TITRE: DU DISCOURS HORS-NORMES D'UN TUEUR EN SÉRIE À LA RECONSTRUCTION DISCURSIVE DE SOI ET DE L'ESPACE ÉPISTOLAIRE : UNE NORMALITÉ REVENDIQUÉE ET RÉGÉNÉRÉE

Auteur(s): Olga Galatanu, Professeur Émérite en Sciences du Langage, LABoratoire CoDiRe, EA 4643, UNIVERSITÉ DE NANTES

PUBLICATION: ÉCRITS HORS-NORMES

PAGES: $92-114$

DiRecteurs: Agnès Steuckardt et Karine Collette

ÉdITEUR: LES ÉDITIONS DE L'UNIVERSITÉ DE SHERBROOKE, 2019

ISBN: 978-2-7622-0360-8

URI: HTTP://HDL.HANDLE.NET/11143/15575

DOI: HTTPS://DOI.ORG/10.17118/11143/15575 


\section{Du discours hors-normes d'un tueur en série à la reconstruction discursive de soi et de l'espace épistolaire : une normalité revendiquée et régénérée}

Olga Galatanu, Professeur émérite en Sciences du Langage, Laboratoire CoDiRe, EA 4643, Université de Nantes

Résumé : Dans le cadre théorique de la Sémantique des Possibles Argumentatifs (SPA), à l'interface de la sémantique et de l'analyse du discours, nous nous proposons d'identifier dans un corpus de lettres de tueurs en série les mécanismes sémantico-discursifs d'une dynamique de restauration identitaire. Ce processus permet l'installation, dans le discours épistolaire et hors de ce discours, d'une « normalité » revendiquée et régénérée. Notre hypothèse est que le discours sur soi des criminels condamnés à la réclusion perpétuelle ou pire, discours qui prend place dans des échanges épistolaires à caractère interpersonnel hors toute norme institutionnelle, passe par des opérations énonciatives de désignation et nomination/dénomination, de qualification et discrimination susceptibles de réduire l'écart entre « l'identité criminelle » et une normalité reconstruite; susceptibles aussi d'intervertir les valeurs qui forment le cadre institutionnel de la sanction sociale. Ces opérations sous-tendent un processus de cinétisme discursif de la signification des mots qui désignent la criminalité et le criminel, processus permettant de dire l'inavouable et l'insupportable. Nous faisons également l'hypothèse que ces discours qui recréent un lien non imposé et peu probable entre la prison et le monde extérieur seront marqués par un processus de régénération et de «normalisation » de l'espace épistolaire.

Mots-clés : Sémantique des Possibles Argumentatifs, cinétisme sémantique, reconstruction discursive de soi, dynamique de restauration identitaire, espace épistolaire.

Abstract : In the theoretical frame of the Semantics of Argumentative Possibilities (SAP), at the interface of semantics and discourse analysis, we propose to identify in a corpus of letters of serial killers, the semantic and discursive mechanisms of an identity restoration dynamics. This process allows the installation, in the epistolary discourse and out of this discourse, of a claimed and regenerated "normality". Our assumption is that the discourse on oneself of killers condemned to perpetual reclusion or worse, discourse which takes place in epistolary exchanges of interpersonal matters, out of any institutional standard, implies operations of designation and nomination/denomination, qualification and discrimination, likely to reduce the difference between "the criminal identity" and a reconstructed normality, likely also to invert the values which form the institutional framework of social sanction. These operations underlie a semantic kinesis (process of discursive change of lexical meaning) of words which indicate the criminality and the criminal. This process makes possible to say the unspeakable and the unbearable. We also make the assumption that these discourses which recreate a not imposed and less probable link between the prison and the external world will be marked by a process of regeneration and "normalization" of the epistolary space.

Key words: Semantics of Argumentative Possibilities, semantic kinesis, discursive reconstruction of oneself, identity restoration dynamics, epistolary space. 


\section{Introduction}

Dans une perspective théorique à l'interface de la sémantique et de l'analyse du discours, la Sémantique des Possibles Argumentatifs (désormais la SPA), nous nous proposons d'identifier dans un corpus de lettres de tueurs en série ${ }^{1}$, les mécanismes sémantico-discursifs de recréation d’un univers référentiel autour du statut de hors-normes du criminel, d'une normalité revendiquée et d'un espace épistolaire régénéré, dans cet échange non imposé et peu probable entre la prison et le monde extérieur².

Deux interrogations concernant la catégorie de « discours hors-normes », se trouvent à l'origine de cette recherche. La première porte sur la dimension normative spécifique de chaque champ de pratique sociale et humaine et des discours qui y prennent place, dans différents contextes culturels et linguistiques. La transgression des protocoles qui sous-tendent la production-interprétation du sens discursif, correspondant à des usages partagés par une communauté de sujets parlants ou imposés à ces sujets parlants par des institutions, serait l'indice d'un discours hors-normes, inacceptable dans et par la communauté. Linguistiquement parlant, le repérage de la transgression de cette normativité, se situe au niveau du DIRE au sens de Ducrot (voir par exemple Ducrot, 1984), et donc aussi au niveau du protocole des différents types de contrats de communication, au sens de Charaudeau (Charaudeau, 1991, 1993)³. Ce repérage concerne également le respect du protocole de politesse (Brown et Levinson, 1987), ou d'impolitesse (Culpeper, 2011, Charaudeau, 2014), en usage dans le cadre de ces contrats de communication.

La seconde interrogation porte sur la nonacceptabilité du sens produit dans et par des discours non respectueux des usages sociaux et des systèmes de valeurs partagés par une communauté de sujets parlants, communicants et interprétants. Le repérage des transgressions qui déclenchent l'interprétation du discours comme inacceptable du point de vue du « sens commun »(Sarfati, 2008, Longhi, 2011), sens construit dans l'inter-discours qui fonde le « politiquement et moralement correct », se situe :

- $\quad$ soit au niveau des opérations linguistiques de désignation, de nomination (Siblot, 1997, 2001, Longhi, 2011, Veniard, 2013), ou du processus de dénomination en cours (Kleiber, 1984, 2001, 2003, Galatanu, 2006, 2013) et de qualification du fait social et des valeurs qui lui sont attribuées, ou tout au moins de la non correspondance entre ces valeurs et les faits évoqués ${ }^{4}$ (Galatanu, 2000);

- $\quad$ soit au niveau de la structuration de la dimension dialogique, produisant ainsi de véritables zones de repli monologique (Paveau, 2010).

Le cas des lettres de tueurs en série, condamnés, aux États-Unis, soit à la réclusion perpétuelle soit à la peine capitale (comme ici), lettres adressées à une personne qu'ils ne connaissent pas, pour établir une relation «sans espoir », est doublement intéressant 5 .

1. Furio Jennifer, 1998, The Serial Killers Letters. A Penetrating Look Inside the Minds of Murders, Philadelphia, The Charles Press Publishers.

2. L'article s'inscrit dans un projet à deux voix, puisque parallèlement à notre étude, Abdelhadi Bellachhab étudie les lettres de Robin Gecht, participant au même groupe de tueurs, les « Chicago Ripper murders »).

3. Pour le concept de contrat de communication voir aussi Ghiglione, 1986.

4. Il s'agit d'une non correspondance qui peut être repérée et analysée linguistiquement (voir l'analyse proposée dans la section 2 de cet article).

5. Nous restons ici dans le cadre d'une recherche en intelligibilité, scientifique, sans oublier pour autant l'impact social d'une pareille recherche, son éventuelle implication citoyenne et les possibles recherches finalisées qu'elle rend possibles. 
D’une part, les sujets énonciateurs qui écrivent ces lettres ont un statut « hors-normes » et leurs discours s'inscrivent dans un cadre non normé. A priori, ils peuvent faire preuve de violence verbale, d'agressivité vis-à-vis de leur destinataire, en l'occurrence une jeune femme ${ }^{6}$, avouer l'inavouable et même fabuler ${ }^{7}$, reconnaissant des crimes dont ils ne sont pas responsables, ni accusés, ni reconnus coupables, voire reconnaître des choses inavouables et s'ériger en défenseurs du « droit au crime »

Edward, qui a avoué sa culpabilité, tout en niant sa responsabilité au niveau de la prise de décision du passage à l'acte criminel, et qui écrit depuis le couloir de la mort, propose « un contrat de communication », en réponse à celui sollicité par son interlocutrice:

"I am writing to you because I am interested in finding out what happened to you how you ended in up in prison. [...] I would like to hear your side of the story. Also, please rest assured that I'm an open minded person and that I have no interest in judging you. In my opinion, honesty is all important. It can set you free. It can help you live with yourself. » (Furio, 1998: vii).

L'acceptation de l'échange épistolaire interpersonnel par Edward est ainsi conditionnée par la transparence de son objectif (pas d'interférence avec d'autres types de communication, par exemple journalistique), et par la sincérité des deux scripteurs et même par le dévoilement des sentiments et du vécu le plus intime de chacun:

"You say you would like to know me better. I have no problem with that? But understand only one thing. I am going to be myself. You will not have any games played, and I don't want none played on me. [...] How about you? Besides writing with Honest Killers, What do you do? » (Spreitzer, June, 10/11/97, in Furio, 1998:4)

D’autre part, ces lettres représentent, selon nos hypothèses, les outils de médiation d'une reconstruction discursive de soi pour soi et pour autrui ${ }^{9}$ et de son monde, dans un environnement de l'action langagière à résonance morale, convoquant les préconstruits culturels (Collette, 2013 : 84) d’une « normalité » perdue.

Par ailleurs, Edward laisse transparaître, dès la première lettre qui veut imposer la transparence et la sincérité totale, sa peur de nuire ainsi à son image devant les autres, et même, et tout d'abord, de mettre en danger ses démarches pour obtenir la commutation de sa peine en réclusion perpétuelle:

"Jenny, I am at this still working on my appeals, this does not sound so hot with me. This could hurt me big time. You want me to be Honest. I might be able to get off Death Row, and I really want to. I don't need anything bad of me going around right now. I don't know what you read. But if it is good that you don't judge me.»

(Spreitzer, June-10/11/1997, in Furio, 1998: 4).

6. Voir les « lettres anonymes » à la fin du recueil de Furio (Furio, 1998 : 293-301).

7. C'est le cas de Jeff Libby dont les lettres deviennent le cadre d'un « aveu total » de sa responsabilité et de sa culpabilité (Furio, $1998: 227-237)$.

8. C'est le cas des lettres de David Gore, faisant partie du même recueil de lettres de tueurs en série (Furio, 1998), qui construit une véritable « théorie du crime»(183-224).

9. Voir à ce sujet, Barbier et Galatanu, 1998 : 45-70. 
Tout se passe comme si, dès le début, Edward plaçait la destinataire de ses futures lettres devant une injonction paradoxale : d'une part, échanger dans un espace épistolaire interpersonnel reconstruit comme un espace normal d'échange sincère, entre deux personnes fondant une relation de confiance, voire d'amitié, et d'autre part, accepter l'inacceptable et le reconstruire également comme acceptable, ou tout au moins supportable, et de ce fait conforme à sa propre dynamique de restauration identitaire.

Nous allons définir d'abord notre cadre conceptuel et avancer, dans ce cadre, deux hypothèses (section 1 de cet article), que nous nous proposons d'illustrer et de conforter par notre analyse des lettres d'Edward. La section 2 sera consacrée à la construction discursive des images et de la dynamique identitaire d'Edward, dans le cadre conceptuel et dans la perspective théorique en sémantique linguistique que nous évoquons dans la section 1. Enfin, la section 3 sera consacrée à la reconstruction d'un espace épistolaire régénéré. Quelques conclusions sur les fonctions identitaires de l'espace épistolaire nous permettront d'envisager des pistes de recherche comparative des discours des incarcérés dans différents contextes socioculturels, d'une part et d'autre part, de ces fonctions (d'une communication à distance et en différé) et des fonctions de l'entretien (interaction orale et en présence) avec les incarcérés dans le même contexte socioculturel. Nous pensons ouvrir ainsi également cette recherche vers une recherche appliquée à la vie sociale et impliquée en elle, et tracer des pistes de recherche finalisée à portée citoyenne.

\section{Cadre conceptuel et hypothèses sur un discours hors-normes : les lettres d'Edward}

Dans cette première section, nous aimerions expliciter, même rapidement, nos points de vue observationnels sur le sujet humain et social, sur le sens et la signification linguistique et sur les liens qui existent entre les champs de recherche qui en font leur objet.

La réflexion menée sur les liens entre ces deux champs de recherche nous conduit à poser un cadre conceptuel d'interface, à la fois interdisciplinaire et intra-disciplinaire : cadre conceptuel pour définir l'identité et les dynamiques identitaires (1.1.) ; cadre conceptuel pour appréhender la construction du sens discursif et la reconstruction de la signification linguistique et, ce faisant, la construction et la reconstruction, dans et par le discours, d'images identitaires de soi pour soi, de soi pour autrui et de son environnement (1.2.).

\section{Identités et dynamiques identitaires}

Nous avons proposé ailleurs (Galatanu, 1996, 2010b) une définition sémantico-discursive de l'identité, que nous reprenons ici pour la confronter (et la conforter) à des définitions qui nous semblent construire une filiation constructiviste du sujet humain et de ses dynamiques identitaires:

Dans cette perspective, je propose une définition de l'identité comme une construction mentale et discursive de soi et d'autrui, dans des contextes situationnels et cognitifs ayant pour enjeux les relations et les interactions des sujets parlants. Sur le plan linguistique, cette construction passe par des opérations énonciatives : désignation, dénomination, définition, qualification et discrimination. (Galatanu, 2010b : 125). 
Ce point de vue observationnel du phénomène identitaire s'inscrit, pour l'essentiel, dans la filiation des approches constructivistes d'une identité « plurielle », « en construction » (Létourneau, 1994: IX), qu'il s'agisse d'approches en analyse du discours (Deshaies et Vincent (dir.), 2004), ou en ethnolinguistique (Riley, 2007), en sciences de la communication (Tracy, 2002), ou en sociologie (Dubar, 1991, 1996, Pascual, 1997). Nous aimerions rappeler ce qui nous paraît être représentatif de cette filiation et préciser ainsi ce cadre conceptuel de l'appréhension du phénomène identitaire. Dans son article sur « La construction de l'identité », l'anthropologue Dorais (Dorais, 2004) souligne le fait que l'identité fait l'objet depuis plusieurs décennies de nombreuses études et définitions dans les Sciences Humaines et Sociales. Ce phénomène humain et social relève certainement des champs de recherche divers dans de nombreuses disciplines: l'anthropologie, l'ethnologie, la sociologie, la psychologie sociale, la philosophie, la sociolinguistique, la pragmatique, en général et en particulier l'analyse linguistique du discours ${ }^{10}$.

Dans l'article mentionné, Dorais synthétise son point de vue en trois éléments qui sous-tendent une définition anthropologique susceptible de faire apparaître le rôle fondamental du « discours langagier » dans la construction identitaire (Dorais, 2004 : 3-4) : l'identité est un rapport (personnel ou collectif) construit par l'homme (ou par une communauté humaine) avec l'environnement, l'identité est donc construite; l'identité équivaut à la relation qu'on construit avec son environnement, appréhendé comme l'ensemble des éléments signifiants faisant partie de l'entourage d'une personne, et par extension, d'une collectivité.

Ce point de vue rejoint ainsi plusieurs approches discursives proposées pour l'étude des identités et de nombreuses analyses dans cette perspective. À titre d'exemples, on peut citer les ouvrages collectifs /dentités sociales et discursives du sujet parlant (Charaudeau (dir.), 1991), Discours et constructions identitaires (Deshaies et Vincent (dir.), 2004), Constructing Identity in interpersonal communication (Palander-Collin, Lenk, Nevala, Sihvonen et Vesalainen (eds), 2010). Pour notre part, nous avions proposé ailleurs également une approche discursive de l'identité (Galatanu, 1996) qui inscrit notre démarche dans cette vaste filiation. Cette démarche s'est précisée dans une perspective sémantico- discursive, avec les travaux sur l'identité dans le cadre théorique de la SPA (par exemple, Galatanu, 2009a, 2010a, b ; Prieto Guzman, 2015).

Nous reprenons ici la définition anthropologique de l'identité proposée par de Dorais, pour la commenter et la conforter avec des éléments issus à la fois d'une approche relevant de la sociologie de l'éducation et d'une approche en Sciences du Langage (sociolinguistique, mais aussi sémantico-discursive) :

Chaque individu possède sa propre conscience identitaire qui le rend différent de tous les autres. Cela signifie que l'identité est appréhendée comme phénomène individuel. On peut fondamentalement la définir comme la façon dont l'être humain construit son rapport personnel avec l'environnement. (Dorais, $2004: 2$ )

10. Les autres disciplines des Sciences du Langage semblent moins concernées par la problématique de la construction identitaire, mais dans le modèle théorique qui est le nôtre, la SPA, la sémantique est non seulement convoquée, mais elle joue un rôle central dans l'analyse de la reconstruction identitaire. 
Dans l'article cité, Dorais traite aussi des « identités collectives », en précisant:

[...] qu'une bonne partie des rapports que l'humanité entretient avec son environnement sont modelés par les actions et les représentations des sociétés auxquelles hommes et femmes appartiennent et qui, dans notre univers en voie de mondialisation, voient leurs frontières s'élargir constamment. Les identités sont donc aussi collectives puisqu'elles sont largement partagées par des groupes d'individus. (Dorais, $2004: 4$ )

Pour notre part, nous retenons le caractère relationnel et dynamique du phénomène identitaire, en perpétuel mouvement selon les aléas de l'environnement, éléments congruents, nous sembletil, avec notre propre description de l'identité, comme une configuration représentationnelle d'images en interaction (y compris langagière) avec autrui et en permanente reconstruction : de soi pour soi, de soi pour autrui, représentation de l'image que les autres se font de soi et image de soi idéale (Barbier et Galatanu, 1998: 65-69).

Nous ajoutons à cette description du phénomène identitaire au moins 4 éléments issus de nos travaux antérieurs et de notre réflexion sociolinguistique et sémantico-discursive :

1. La construction identitaire produit une diversité d'images identitaires qui s'accompagne néanmoins de la construction identitaire unique, support de toutes les expériences, souvent désignée en termes de personnalité. (Barbier et Galatanu, 1998 : 58)

En termes langagiers, le discours sur soi (pour soi et pour autrui) propose donc un noyau commun aux différents avatars des différentes représentations du sujet en action (social, familial, professionnel et, pour notre étude, avant les actes criminels, dans l'acte criminel, après la condamnation qui change le statut social, voire même humain du sujet, par le passage de « la normalité » à « la hors-normalité »).

2. Cette construction identitaire, qui est un processus continu en lien avec les changements de l'environnement et des expériences, a une dimension cognitive-affective, résultat du processus d'intériorisation des expériences du sujet, les deux aspects qui « modalisent » la représentation de soi et d'autrui étant inséparables (Damasio, 1994).

3. Dans les transformations émotionnelles et plus généralement dans la transformation de soi en action, selon les évolutions de l'environnement, et encore plus quand il s'agit de l'appartenance à une collectivité (dimension culturelle, nationale ou ethnique de l'identité), le rôle du langage est central et cette centralité est objet de réflexion aussi bien des sociologues (Bourdieu, 1982), des philosophes (Ricoeur, 1990), des linguistes (Benveniste, 1966, Galatanu, 1996, 2010b, Vincent, 2000, Collette, 2004) que des psychologues (Lutz, 1982, Averill, 1982, Averill et Rodis, 1998 ) et des anthropologues (Anderson, 1996, Dorais, 2004).

4. Le discours est une voie d'accès privilégiée vers les images identitaires de soi et d'autrui, mais également un facteur, un déclencheur de transformation de soi, par et dans l'univers référentiel, toujours renouvelé qu'il propose (Galatanu, 2009b).

\section{Le cadre théorique de la SPA au service de l'étude de l'identité}

La Sémantique des Possibles Argumentatifs représente un cadre théorique à l'interface de la sémantique linguistique et de la pragmatique, de la signification linguistique et du sens construit dans et par le discours. Cette interface n'est pas appréhendée en SPA comme une « jonction », ni même comme une intégration de la pragmatique à la sémantique permettant d'analyser « les mots du discours » et plus largement tous les 
mots du lexique d'une langue au niveau de leur potentiel discursif, mais comme un espace de deux formes de manifestation simultanée du même phénomène linguistique (Galatanu, 2013).

Cette approche ${ }^{11}$ est fondée sur l'idée que chaque occurrence discursive propose, en même temps que le sens discursif en cotexte et en contexte, une signification lexicale pouvant correspondre à celle stabilisée à un moment donné pour une communauté linguistique, mais pouvant aussi être reconstruite, «régénérée » (pour emprunter à Bellachhab (2012) un terme plus saillant), par le discours.

Quatre assertions de la SPA, l'habilitent pour l'analyse de la construction / régénération / reconstruction des images identitaires de soi et d'autrui et des dynamiques identitaires.

(a) La signification des mots est envisagée comme une construction ou une (re)-construction permanente à partir du sens proposé par les occurrences discursives. Ceci revient à dire que la signification est porteuse de l'expérience individuelle et de l'expérience discursivement partagée du monde dans une culture donnée et de ce fait la SPA est une approche sémantique argumentative mais également cognitive. Pour ce rapprochement, cette alliance entre la SPA et la sémantique cognitive de Langacker (2008), nous renvoyons aussi à Bellachhab (2012, 2013).

Dans une dynamique de restauration identitaire (cf. Barbier et Galatanu, 1998: 67), la tentative de normalisation identitaire sera porteuse sur le plan des observables dans le discours, de qualifications (fonction attributive, identificatoire), de discriminations (fonction discriminatoire) et d'affirmations d'appartenance, d'adhésion à un groupe humain (fonction classificatoire) (Galatanu, 1996 : 51), notamment à ce qui est perçu comme caractérisant la normalité dans une communauté culturelle, au sens le plus large du terme et ipso facto, linguistique, inscrite de façon largement partagée et relativement durable dans la signification « des mots pour la dire».

(b) La description de la signification lexicale est susceptible de rendre compte du degré de stabilité des associations de représentations qu'elle porte (dont celles du contexte discursif où le sens se construit), ces associations pouvant être contraintes par la situation de communication et / ou par le cotexte.

Nous avons ainsi une base proprement linguistique, de nature sémantique, pour rendre compte du degré d’individuation de la construction identitaire des énonciateurs-scripteurs qui nous intéressent ici.

(c) La description de la signification linguistique rend compte du niveau d'inscription des valeurs modales qu'elle porte: le noyau, comprenant les propriétés essentielles, identitaires de cette signification (crime, murder, killing - nominal, dont les noyaux sont porteurs de valeurs axiologiques morales et éthiques négatives et de la valeur déontique <interdit>), ou les stéréotypes, ensembles ouverts d'associations des éléments du noyau avec d'autres représentations, ancrés culturellement et de ce fait évolutifs, cinétiques (par exemple « souffrance » pour killing ou murder, ou encore « regret », « excuses », ou « pardon»).

11. Voir, en particulier, Galatanu, 2009b, 2013 et des travaux doctoraux dont les références se trouvent dans Galatanu, 2013. 
À partir du noyau et des stéréotypes, on peut générer des enchaînements discursifs (à visée axiologique, donc argumentatifs), virtuels, potentiels - les Possibles Argumentatifs (PA) et les confronter avec des enchaînement-occurrences, les Déploiements Argumentatifs (DA), ou Discursifs (DD), qui peuvent être conformes aux PA, conformistes par rapport au protocole sémantique du mot, ou au contraire, non conformes, déclenchant une reconstruction de la signification lexicale, ne serait-ce que le temps de l'occurrence discursive.

Pour confronter les PA et les DA/DD, nous pouvons adopter deux démarches dans nos analyses, selon le sens du rapport PA - DA (DD), celle adoptée dans cette recherche étant de partir des DA/DD, c'est-à-dire des mots mobilisés dans le discours et du sens qui leur est attribué par le discours, pour les comparer avec la signification reconstruite d'après les discours lexicographiques. Ces derniers sont envisagés ici comme des discours « experts » pour une communauté linguistique à un certain moment historique.

\section{Hypothèses sur un discours hors-normes : les lettres d'Edward}

Dans ce double cadre théorique, nous avançons deux hypothèses que nous nous proposons d'illustrer et confirmer par l'analyse des 11 lettres d'Edward qui forment notre corpus ${ }^{12}$. La première hypothèse, plus générale, porte sur la fonction identitaire complexe de ce type d'échange épistolaire hors-normes. La seconde concerne la régénération de l'espace épistolaire même à travers la présentation de soi lorsque celleci s'inscrit dans une dynamique identitaire de restauration de soi, comme c'est le cas d'Edward ${ }^{13}$.

Hypothèse 1 : Nous avançons l'hypothèse que le discours hors-normes des lettres des tueurs en série incarcérés, lettres adressées à une correspondante inconnue et visant à construire une relation « sans espoir », joue une fonction non seulement de médiateur de la reconstruction identitaire, mais également et surtout celle de déclencheur de la dynamique de restauration identitaire et de la régénération des images de soi pour soi et pour autrui, pour rétablir une correspondance de ces images avec les images de soi « idéales » ou « idéalisées », attendues par le destinataire dans son contexte culturel.

Le corollaire proprement linguistique de cette première hypothèse est que la construction discursive de ces images identitaires correspondant à des préconstruits culturels ${ }^{14}$ passe par des mécanismes sémantico-discursifs de reconstruction de la signification des mots désignant les actes criminels et l'acteur même de ces actes: opérations de nomination pouvant aller jusqu'à la modification du lien dénominatif, et donc des propriétés essentielles, nucléaires des significations de ces mots.

Hypothèse 2: Nous avançons également l'hypothèse que la dynamique discursive de restauration identitaire, dans un environnement où la mise en dehors de la société de l'auteur des crimes s'appuie sur l'aveu

12. Nous ne prétendons pas valider ces hypothèses et généraliser les résultats à partir d'un corpus si limité, mais nous pensons plutôt pouvoir conforter nos hypothèses et les outils investis dans l'analyse pour entreprendre l'étude de l'ensemble des lettres publiées par Furio et d'autres témoignages recueillis dans nos futures enquêtes auprès d'incarcérés en France et dans d'autres pays, prévue dans le cadre d'un projet en cours d'élaboration.

13. Nous avons analysé ailleurs, sous un angle différent, le cas des lettres d'un autre incarcéré sollicité par Furio, Harvy, qui s'inscrit dans la même dynamique identitaire. En revanche, nous n'y avons pas abordé la fonction méta-discursive, de reconstruction de l'espace épistolaire, l'objectif de cette première recherche sur les incarcérés étant de comparer la dynamique identitaire de Harvy et celle d'un incarcéré français condamné, en France, également à la réclusion perpétuelle. (Voir Galatanu, 2010b : 125138).

14. Voir Collette, 2013. 
même de cet auteur, s'accompagne nécessairement d'une dynamique de reconstruction discursive de l'espace épistolaire hors-normes.

Le corollaire proprement discursif de cette hypothèse est que cette reconstruction de l'espace épistolaire passe d'une part, par des opérations énonciatives conformes au protocole de politesse de la communication épistolaire dans un contexte culturel donné (formules d'adresse, d'ouverture et de clôture, partage de l'espace de chacun des participants à la communication), et d'autre part, par une modalisation axiologique positive (affective, hédonique, morale, intellectuelle, voire même esthétique) ${ }^{15}$ incidente au sujet énonciateur, très explicite et très forte, permettant la construction d'un éthos idéal pour l'échange épistolaire interpersonnel.

\section{La construction discursive des images et de la dynamique identitaire d'Edward}

\section{Les actes : dénomination, nomination ou reconstruction discursive du crime ?}

Conformément à l'hypothèse 1, Edward, qui a avoué l'inavouable et dont le discours est censé réécrire ses actes, tout au moins ceux qu'il assume, pour les rendre acceptables, va déployer dans son discours, des stéréotypes non conformes à ceux portés par «les mots de la criminalité », proposant ainsi par des mécanismes sémantico-discursifs que nous avons regroupés sous le nom de «stéréophagie » (Galatanu, 2009a : 189-2009), une atténuation des valeurs axiologiques négatives portées par ces mots, comme dans l'exemple 1.

(1) «When all these murder's happened, I was sick all the time, see I pass out when I see blood, it has been like that all my life, even in here, when the Doctor takes blood, I am on the floor. When I get a paper cut and I see blood, I am on the floor. It don't matter how much blood, I will pass out. » (Letter : 7).

L'étude des mots d'Edward pour dire ses actes, qui l'ont conduit vers le couloir de la mort, fait apparaître plusieurs spécificités de la reconstruction discursive de sa vie d'avant les aveux, la condamnation et l'incarcération:

On constate d'abord une prédominance de désignations pronominales, neutres du point de vue axiologique : total 31 occurrences : it (7) - Exemple 2, this/all this/all of this (10) - Exemple 3, what I did/ done/ have done (10) - Exemple 4, what I was forced to do (1), what he (Robin) wanted (1), what others do (1), what has happened (1) - Exemple 5.

(2) «I guess that is why / went to the police, to once and for all to stop it».

(3) «I was 21 year's of age when this started.»

(4) « I don't feel proud for what I did. »

(5) «I do feel bad for what has happened »

15. Pour la définition des valeurs modales voir Galatanu, 2000, Cozma, 2009. 
Nous aimerions souligner deux éléments qui jouent un rôle fondamental dans la restauration discursive de l'identité d'Edward: la présence d'une forme impersonnelle, what was happened, qui annule toute responsabilité pour les actes avoués et de deux occurrences qui dissocient la responsabilité (la prise de décision et la préméditation) de la mise en œuvre de l'acte criminel ; le déploiement répétitif de ces éléments des stéréotypes, qui acquièrent ainsi le statut d'éléments saillants, a une fonction d'atténuation de la valeur modale négative du noyau des mots qui désignent l'acte, comme dans les exemples 6, 7, 8, 9 :

(6) «I would have loved to write to the families [of the victims] and let them know how bad and sad I feel of what I had done. »

(7) « Jenny, I do know nothing can bring back the Victim's. I wish I really could. »

(8) «I know you are glad to hear me speak of how I feel of the Victims. »

(9) «Jenny, in the eye's of God, I have been forgiven for my crimes. »

L'exemple (9) est le plus intéressant car il déploie une association stéréotypique de la signification de crime, mot porteur d'une valeur déontique inscrite dans son noyau même, <interdit par la loi>, Dieu intervenant dans la restauration identitaire d’Edward que la société lui refuse.

L'inventaire des nominaux et des verbes utilisés pour désigner les actes d'Edward ou les nier (28 occurrences au total), fait apparaître une faible représentation des mots les plus chargés de valeurs axiologiques morales négatives et de valeurs déontiques: crimes (4 occurrences), killing(s), to kill (6 occurrences) murder (2 occurrences). Leur cotexte et donc le déploiement de leur potentiel négatif est aussi au service du rétablissement d'une normalité régénérée d’Edward. Deux des occurrences de crimes sont présentées à travers les questions des enquêteurs (exemple 10) et une occurrence déploie une association conforme au protocole sémantique du mot, mais transgressive, en «Pourtant » (au sens de Ducrot, 1995, Galatanu, 2000). Killing et to kill, apparaissent 5 fois dans des structures négatives qui établissent la nonresponsabilité d'Edward par rapport aux actes dont il est coupable (exemples 11,12) et une fois dans une structure impersonnelle (exemple 13).

(10) «I had gun's put in my face to force me to do the crime's. »

(11) «I am not one to hurt women, or even kill women. »

(12) "Robin's locked up for one murder. [...] I was so happy for that. All this was over. No more killings. »

(13) « No, you have not forced me to talk about the killings, I did that on my own [...]. »

Sur une échelle graduelle de valeurs morales négatives, mais porteuses par leur noyau de valeurs hédoniques affectives négatives, les mots hurt (women) et hitting apparaissent 3 fois, dans le même type de structure négative que précédemment. Les résultats des crimes sont désignés par les mots bodies, en structure passive et victims, dans un environnement sémantique déployant un faisceau de stéréotypes rétablissant la normalité d'Edward : compassion, regret, excuses, remords, souffrance, comme dans l'exemple 14.

(14) « Jenny, I do know nothing can bring back the Victim's, I wish I really could. » 
Enfin, il y a dans l'inventaire des expressions mobilisées pour reconstruire discursivement ses actes, le nominal thing ou le groupe nominal bad things ou encore to do bad, qui, de par leur degré élevé de généralité (ils couvrent une gamme large d'erreurs, fautes, délits et crimes), occultent la gravité des faits qui ont fait acquérir à Edward le statut de hors-normes et d'incarcéré dans le couloir de la mort.

L'exposition concrète et très crue d'une partie des crimes présente Edward comme impuissant pour intervenir, ou forcé d'agir mal ou enfin, grâce aux structures impersonnelles ou passives qui occultent l'auteur de ces crimes, non impliqué.

\section{L’acteur : une identité régénérée}

Nous préférons utiliser ici le terme de « régénérée », que nous devons à Bellachhab (Bellachhab, 2012), même si nous ne lui attribuons pas le même sens (chez Bellachhab, c'est un synonyme de « reconstruite »), justement parce que l'identité de criminel d'Edward n'est pas niée par celui-ci ou tout au moins elle n'est niée que partiellement : au niveau de la responsabilité du passage à l'acte, au niveau du vécu de ces actes et au niveau d'une partie de ces actes dont il n'avait pas connaissance. Mais cette identité est régénérée, car associée : à des sentiments et des vécus acceptables par la communauté, par un mécanisme de restauration identitaire ; à un véritable rejet, dégoût des actes criminels de son groupe d'appartenance, par un mécanisme de discrimination identitaire ; à une sensibilité qui le place dans une position privilégiée parmi les « gens normaux » ${ }^{16}$, par un mécanisme d’individuation.

La « normalization » d'Edward passe par des operations énonciatives de restauration identitaire par rapport à ces actes passés et de discrimination par rapport au statut de hors-normes: I got sick and passed out, I didn't enjoy killing, Killing was not fun for me, I have much hate for a person like myself, I feel stupid for what I did, Because, like you, I myself feel horrible of how..., Killing was not my way of doing things, I don't feel proud for what I did, I feel very sad for all of this, I can't be happy, lives were taken.

Les deux derniers énoncés, de même que l'exemple (15):

(15) «I am not a Heartless person you read about. But no one else knows this, because no one bothered to try and get to know the real Edward Spreitzer»,

sont particulièrement intéressants parce qu'ils illustrent bien l'itération permanente entre un statut horsnormes après tout assumé, ou partiellement assumé et l'installation d'une dynamique de restauration identitaire qui aboutit à un retour à la normalité : a. «je cherche la bonne personne pour l'épouser » (comme dans le monde extérieur, de la normalité), pourtant b. "ça sera probablement pour 3 ou 4 ans seulement », mais c. « je n'ai jamais eu ou senti l'amour d'une femme qui soit bonne », donc a. « je cherche la bonne personne pour l'épouser », comme dans le monde extérieur, de la normalité (schéma 1).

16. Voir la typologie des dynamiques identitaires proposée dans Barbier et Galatanu, (1998: 65-70). 
Schéma $1:$ : a pourtant $b$ », mais « b pourtant $c$ », « c donc $a$ ».

\section{Les mots de la criminalité dans le discours d'Edward}

Nous avons analysé ailleurs (Galatanu, 2010b :130) la signification des dénominations d'actes axiologiquement négatifs : crime, délit, faute et erreur, telle qu'elle est proposée par les discours lexicographiques et nous avons inscrit ces mots sur deux échelles des degrés d'intensité (gravité morale et responsabilité juridique). Nous allons proposer ici le même retour de l'étude des DA/DD des mots de la criminalité dans le discours d'Edward vers la construction de la signification de ces mots à partir des discours lexicographiques $^{17}$, donc vers les PA que cette signification habilite. Comme nous l'avons vu précédemment, parmi les désignations des actes d'Edward, nous rencontrons les dénominations : crime(s), murder(s), killing(s) et le verbe to kill. Les schémas 2, 3 et 4 présentent les noyaux et des exemples de stéréotypes, permettant la génération de PA de ces mots.

\section{Schéma 2 : Crime}

Noyau : X DEVOIR NE PAS FAIRE P [interdit] / FAIRE P [obligatoire] PT FAIRE $p$ [interdit] / NE PAS FAIRE P [obligatoire] DC X AGIR TRES MAL ET X MERITER SANCTION

Exemples de stéréotypes: DC punition par la loi/DC lutte contre P/contre Non - P, DC X inspire le rejet des autres...

\section{Schéma 3 : Murder}

Noyau : X DEVOIR NE PAS ENLEVER LA VIE A Y PT X VOULOIR ENLEVER LA VIE A Y DC X PREMEDITER D'ENLEVER LA VIE A Y DONC X ENLEVER LA VIE A Y DONC X AGIR MAL DC X MERITER PUNITION SEVERE

Exemples de stéréotypes : DC punition sévère, DC inspirer l'horreur, DC x hors la loi DC x hors-normes...

\section{Schéma 4 : Killing}

Noyau : X DEVOIR NE PAS ENLEVER LA VIE A Y

17. Nous considérons les discours lexicographiques comme des discours experts « du langage ordinaire ». Ils sont censés rendre compte du partage des représentations sémantiques des mots d'une communauté linguistique et culturelle, à un moment donné. 
PT

X VOULOIR ENLEVER LA VIE A Y (P)

DC

X ENLEVER LA VIE A Y (P)

DC

XAGIR MAL

DC

X COUPABLE de P

ET

X RESPONABLE de P

DC

X MÉRITER SANCTION TRÈS SÉVĖRE
DC

X NE PAS VOULOIR ENLEVER LA VIE A Y (P)

PT

X ENLEVER LA VIE A Y

DC

X AGIR MAL

DC

X COUPABLE de P

PT

XNON RESPONSABLE de P

PT

X MÉRITER SANCTION SÉVÈRE

Où DC = Donc et PT = Pourtant.

Sur l'échelle de gravité que nous avons proposée (Galatanu, 2010b : 130), murder est le plus grave sur le plan moral aussi bien que juridique, killing est grave sur le plan juridique, mais moins grave que murder sur le plan moral et crime est le moins grave sur le plan moral et juridique, car il couvre, certes murder et killing, mais aussi d'autres transgressions de la loi, moins graves.

\section{Schéma 5}

Droit : $-/+$ crime $<+$ killing $<++$ murder

Morale +/ - crime $<-/+$ killing $<+$ murder

Où : « - symbolise le pôle axiologique négatif et «+»= une atténuation de la valeur négative.

L'analyse des dénominations de l'acte TO KILL (TUER), très peu nombreuses par rapport aux formes pronominales de désignations ou aux génériques thing, bad thing, fait apparaître l'absence d'une prise en charge de murder:

(16) «When all these murder's happened, I was sick all the time»

(17) «Robin's only locked for up for One murder.

Killing apparaît quatre fois dans des énoncés qui nient la responsabilité et même la capacité d'accepter ces actes d'Edward et une fois dans une structure impersonnelle (exemple 13 cidessus). Crimes apparaît deux fois, la deuxième fois avec l'adjectif possessif (my crimes) pour désigner des actes assumés par Ed- 
ward, mais avec un déploiement discursif transgressif (exemple 18) ou une association stéréophage (le pardon de Dieu) qui bloque les autres déploiements, axiologiquement négatifs (comme dans l'exemple 9).

(18) "When I woke up, I was still in the Van in back of Robin's house. I understand her body was not found. And I am locked up for the crime» (letter 5).

Dans le monde discursif d’Edward, la culpabilité atténuée par ses désignations génériques ou déployant des éléments de la signification des dénominations et des désignations des actes qui, sans remettre en cause la gravité des faits, reconstruisent l'identité de l'auteur, la responsabilité niée, rejetée, la sensibilité affichée, les projets de vie normale envers et contre tout, construisent une normalité retrouvée dans l'échange épistolaire.

\section{La reconstruction discursive d'un espace épistolaire régénéré}

La dynamique de normalisation de l'identité d'Edward s'accompagne d'un processus de normalisation du discours épistolaire hors-normes. Elle est énoncée, d'une certaine façon, dès le début dans un mouvement de va et vient entre la normalité de l'interaction verbale, proposée par l'interlocutrice potentielle et la situation hors-normes de l'incarcéré :

Tableau 1

\begin{tabular}{|l|l|l|}
\hline Normalité & mais & Anormalité \\
\hline I love to write & but & The problem is finding someone to write to \\
\hline I do have a few pen friends & but & They don't know of me being locked up \\
\hline I don't feel good about that & but & I am so lonely \\
\hline $\begin{array}{l}\text { You say you would like to know me better and } \\
\text { I have no problem with that, so I am going to } \\
\text { be myself }\end{array}$ & but & $\begin{array}{l}\text { I don't need anything bad of me going } \\
\text { around right now }\end{array}$ \\
\hline It is good that you don't judge me & but & I don't know what you read \\
\hline
\end{tabular}

Nous souhaitons présenter ici trois éléments qui forment le cadre de la normalisation du discours épistolaire : la présentation de soi et les salutations, les formules d'adresse, d'ouverture et de clôture de la communication épistolaire, la signature. D’autres phénomènes et mécanismes sémantico-discursifs, comme la modalisation incidente au sujet énonciateur, au destinataire, ou aux objets de leurs propos, participent à la normalisation du discours épistolaire, mais nous ne pouvons pas les aborder dans l'espace réduit de cet article.

\section{La présentation de soi}

La présentation de soi dans la relation épistolaire convoque des valeurs positives, même lorsqu'il s'agit d'assumer son statut hors-normes (il dit appartenir à la catégorie des « honest killers », être capable d'être honnête et être soi-même). L'ethos se construit autour de ses qualités humaines, activées ou à activer dans cette relation : I may be a big person, I am very much gentle, I am not one to hurt women, but men are so really stupid out in the free world, You say I am Sweet and Human and people need to see that, I don't have no respect for a man who does harm to a woman and kids, I am known on this unit to help others in finding 
some great deals, You say I have so much personality and so much to give, My personality has always been the same, I would love to have a family. These men have, and now they want out, I am nothing but a Big Teddy Bear, I could carrie you in my arm's all day long, I would be ever so Gentle.

La normalisation du discours épistolaire, faisant écho à la normalisation identitaire prend également la forme d'un équilibre entre l'ethos et le pathos, entre la construction de l'identité énonciative et celle du destinataire, très présent dans le texte d’Edward (voir la section 3.2.).

\section{Les formules d'adresse et l'acte phatique SALUER}

Les formules d'adresse et les salutations posent dès la première lettre une relation interpersonnelle non formelle et amicale, et ipso facto la reconnaissance de la personne inconnue qui a déclenché l'échange, comme possible partenaire à cet échange, qui est d'ailleurs, comme nous l'avons montré, «contractualisé » comme sincère et sans secrets, fussent-ils intimes.

\section{Les formules d'adresse et d'ouverture de la communication}

À l'exception de la lettre 3, qui commence de manière abrupte, étant plutôt une suite nocturne d'une lettre commencée déjà au cours de la journée, on constate le passage du réalisateur linguistique de SALUER Hi à Hello, les deux expressions phatiques ayant le même degré de familiarité. Pour la formule d'adresse, elle évolue du prénom à l'appellatif beautiful, marquant la reconnaissance d'une attitude masculine ouverte vis-à-vis de la jeune femme qui est le destinataire d'Edward et à qui d'ailleurs ce dernier demande avec insistance une photo.

La fonction phatique de l'expression familière qui accompagne la formule d'adresse est renforcée par l'usage d'expressions d'usage courant dans les lettres échangées entre personnes proches (famille, amis, connaissances), toutes faites, voire figées : I am doing ok, I am doing fine et ceci malgré le contexte inadéquat à leur contenu propositionnel (le couloir de la mort, la culpabilité, le regret d'en être arrivé là, les tentatives désespérées de faire changer la sentence).

Nous avons identifié cinq groupes de formules d'ouverture de la communication dont la fonction phatique s'accompagne de l'expression d'actes de langage visant une sorte de connivence sur la normalité de l'échange épistolaire : le reproche si la réponse n'est pas rapide, l'excuse si le retard concerne la lettre d'Edward:

(a) Formules toutes faites, affirmant, au-delà de leur contenu propositionnel figé la normalité de l'échange: I am doing somewhat ok. I could be better (letter 1); I am doing fine and I hope the same for you and your Family (letters 5 et 7), I am doing fine today and I hope the same for you and your family (letter 11).

(b) Formules mixtes : toutes faites, mais accompagnées de l'expression du reproche adressé au destinataire (pour son silence) et de la joie des « retrouvailles » à travers la lettre : I am doing much better, now that I heard from you away by letter, I hope you and your Family is doing well (letter 6); It was so ever good to hear from you. I have so much to write about. But first so you can see I am not dead. And I am fine, and hope the same for you and your Family (letter 10). 
(c) Formules marquées affectivement (reproche et joie): I got your letter yesterday. I was glad to hear back from you [...] (letter 2); I just got your letter and I am first to write back (letter 4).

(d) Formules d'excuses pour son propre silence avec rappel de la situation (la lutte pour changer la sentence, lutte dans laquelle Edward tente d'entraîner son interlocutrice): So sorry for my delay in writing (letter 8) ; Sorry, I fell behind in my writing, because of my case [...] (letter 9).

(e) Expression avec un présupposé de relation d'amitié, voire d'intimité bien acquise: Yes, I went to bed at 3:30 AM writing to you (letter 3).

\section{Les formules de clôture de la communication}

Les formules de clôture marquent, comme celles d'ouverture, une relation et une interaction verbale épistolaires amicales et normales, ou plutôt « normalisées » par le discours d'Edward. Nous aimerions souligner le fait que dans les lettres des autres incarcérés réunies par Furio, les formules sont explicitement plus formelles et plus distantes, correspondant davantage à la situation particulière de l'échange. Cela nous semble confirmer le lien qui existe entre le type de dynamique discursive de restauration identitaire, par l'expression de l'empathie et plus généralement de la sensibilité et des affects, et la restauration d'un espace épistolaire dans la zone de l'amitié et des affects partagés.

Nous avons classé les expressions utilisées en cinq groupes:

(a) La formule amicale et familière take care (lettres 3, 4 et 7);

(b) La formule amicale et familière take care accompagnée d'un acte de langage de demande/ prière : write back, Can I please call you... (lettre 1, qui propose déjà une relation amicale, lettre 5) ;

(c) La formule amicale et familière take care, « enrichie » par une autre expression hédonique affective positive and Smile (lettres 9 et10);

(d) Le remplacement de la formule de clôture par un méta-texte à valeur méta-illocutionnaire ${ }^{18}$ (questionnement sur l'acceptation des aveux par leur destinataire accompagné d'un acte expressif sur la souffrance provoquée par ces aveux et de la promesse de continuer à répondre à la sollicitation des aveux -lettre 6, affirmation d'avoir fait au mieux en avouant et promesse de continuer à le faire-lettre 8) ; cette clôture non conventionnelle et non habituelle concerne justement les lettres dans lesquelles Edward retrace l'histoire qui l'a conduit dans le couloir de la mort.

(e) Enfin, l'expression de l'empathie pour l'éventuel sentiment de solitude de l'interlocutrice et du désir de la «chérir », comme une promesse d'amitié et d'affection, voire de tendresse, clôt la dernière lettre (lettre 11), comme pour marquer définitivement la relation interpersonnelle normale, évoluant de la compréhension à l'amitié et de l'amitié à la tendresse et à la réciprocité dans ce que les partenaires peuvent attendre l'un de l'autre, dans un espace épistolaire régénéré.

Une seule lettre (la lettre 2) se termine sans conclusion, commentaire ou formule de clôture.

18. Pour la définition du «méta-illocutionnaire », voir l'article de Galatanu, Cozma, Fravalo, « Les valeurs sémantico-pragmatiques de "je t’explique", "je m'explique" et les représentations sémantiques des verbes "expliquer" et "a explica” » (2014 : 277294). Voir également, pour la définition de la notion de métadiscours (Garric, 2009). 


\section{La signature}

La signature d'Edward confirme la reconstruction discursive et la « normalisation » de l'espace épistolaire. Elle évolue de l'expression conventionnelle Your truly Edward Spreitzer, par ailleurs adéquate au contrat d'une communication basée sur la totale sincérité, proposé dès la première lettre, jusqu'à la formule amicale, Your Friend always Edward Spreitzer, expression à fonction phatique, mais également à valeur illocutionnaire de promesse, d'engagement vis-à-vis d'une amie (lettres 9, 10, 11). Une étape intermédiaire entre l'expression conventionnelle de la première lettre et l'expression phatique et d'engagement dans l'amitié, est marquée par deux expressions amicales à fonction phatique : Your Friend Edward Spreitzer (lettres 2,4, $5,6,8$ ) et Friends always (lettre 7). Cette dernière signature est fort intéressante, puisque, d'une part, elle ne comporte plus le nom d'Edward, et d'autre part elle inclut le destinataire, Jenny Furio, en tant qu'amie consentante de cette relation, établissant une complicité dans l'univers discursif de l'échange épistolaire.

\section{Conclusion}

L'analyse de la construction discursive des images de soi et de la dynamique identitaire d'Edward a été menée à trois niveaux:

- au niveau de la reconstruction discursive des actes qui l'ont conduit dans le couloir de la mort: l'association discursive des mots pour dire ces actes avec des valeurs affectives et hédoniques négatives, marquant la souffrance et le remords, l'empathie pour les victimes et leurs familles, morales et éthiques positives marquant le rejet de tels actes et pragmatiques négatives, voire même aléthique (impossibilité), marquant le refus d'admettre la performance de ces actes de manière consciente ;

- au niveau de la régénération de l'image de l'acteur de ces actes : par des mécanismes d'association de cette image à des sentiments et des vécus correspondant à des préconstruits culturels de la normalité, par des mécanismes de discrimination par rapport à son groupe d'appartenance (les criminels incarcérés, et plus largement ceux qui font du mal aux femmes et aux enfants), par un mécanisme d'individuation grâce à l'affirmation d'une sensibilité hors commun qui confère une place privilégiée parmi des « gens normaux»;

- à travers la régénération des significations des mots de la criminalité, peu mobilisés et dans un environnement sémantique qui atténue leurs valeurs axiologiques négatives.

Nous pensons avoir montré ainsi que ces lettres hors-normes du point de vue de leur cadre pragmatique, représentent essentiellement des médiateurs, voire même les déclencheurs d'une reconstruction identitaire, notamment de mise en œuvre d'une dynamique identitaire de restauration et d'une dynamique identitaire d'individuation. Cette reconstruction identitaire qui passe par une reconstruction discursive des représentations de soi et du monde (actes et environnement) autour d'une normalité revendiquée et régénérée valide notre première hypothèse.

Nous avons pu voir aussi que cette normalisation de l'univers discursif s'accompagne d'un processus de normalisation du discours épistolaire hors-normes, tout au moins dans le cas étudié, ce qui valide, pour ce cas, notre seconde hypothèse de départ. Les trois éléments qui construisent le cadre énonciatifépistolaire - la présentation de soi, les formules d'adresse et les expressions d'ouverture et de clôture de la communication et la signature- font apparaître un espace épistolaire « normalisé » et une relation interpersonnelle 
amicale, de confiance et de réciprocité dans l'échange, voire même affective, construite dans et par le discours d'Edward.

Les résultats de cette recherche nous conduisent à formuler une nouvelle hypothèse locale (pour le cas étudié) et une hypothèse générale, portant sur les liens qui existent entre la reconstruction identitaire et la reconstruction et normalisation de l'espace épistolaire.

L'hypothèse locale concerne le lien entre la dynamique identitaire mise en œuvre par Edward, de restauration de l'image de soi dans le registre affectif, de la sensibilité et de l'empathie et la normalisation de l'espace épistolaire dans le même registre, de l'affectif, de la confiance et de la complicité amicale, voire affective.

L'hypothèse générale portant sur le discours hors-normes des incarcérés, mis à l'écart de la société, concerne le lien entre le type de dynamique identitaire et l'espace épistolaire que ce discours construit. Ainsi, l'identité régénérée par le discours de Harvy que nous avons étudiée ailleurs ${ }^{19}$, s'appuyant sur une normalité instaurée dans la vie quotidienne en prison, saine et morale, s'opposant à la critique de la vie immorale des gens de l'extérieur, dits « normaux », s'accompagne d'une construction de l'espace épistolaire basé sur une relation plus froide, de prise de distance, voire même de méfiance.

Deux pistes de recherche nous semblent s'ouvrir au terme de ce travail : une recherche comparative à la fois de l'ensemble des discours hors-normes réunis dans le recueil qui nous a fourni le corpus, au niveau de la normalisation des identités et du discours ; une recherche comparative de ce type de discours hors-normes, épistolaire et de données recueillies par des entretiens directs avec les incarcérés, dans des contextes culturels diversifiés (par exemple, en France et au Québec).

19. Voir Galatanu, 2010b. 


\section{Bibliographie}

Anderson Benedict (1996), L'Imaginaire national, Paris, La Découverte.

Averill James (1982), Anger and aggression: An essay on emotion, New York, Springer-Verlag.

Averill James et Pano Rodis (1998), «Le rôle du langage dans les transformations émotionnelles », J.-M. Barbier et O.Galatanu (dir.), Action, affects et transformation de soi, Paris, PUF, 19-43.

Barbier Jean Marie et Galatanu Olga (1998) « De quelques liens entre action, affects et transformation de soi », J.M. Barbier et O. Galatanu (dir.) Action, affects et transformation de soi, Paris, PUF, 45-70.

Bellachhab Abdelhadi (2012), Genèse d'une sémantique conceptuelle au service de l'interaction verbale: des représentations aux réalisations et des réalisations aux représentations, Mémoire de HDR, Université de Nantes.

Bellachhab Abdelhadi (2014), «Les déploiements discursifs, partie émergée de la conceptualisation », A.-M. Cozma, A.Bellachhab et M.Pescheux (dir.) Du sens à la signification: de la signification au sens. Mélanges offerts à Olga Galatanu, Berne, Peter Lang, 367-387.

Beneveniste Émile (1966), Problèmes de linguistique générale, Paris, Gallimard.

Bourdieu Pierre (1982), Ce que parler veut dire. L'économie des échanges linguistiques, Paris, Fayard.

Brown Penelope, Stephen Levinson (1987), Politeness. Some Universals in Language Usage, Cambridge, Cambridge University Press.

Charaudeau Patrick (1991), « Identité sociale et identité discursive. Un jeu de miroirs fondateur de l'activité langagière », P.Charaudeau (dir.) Identités sociales et discursives du sujet parlant, Paris, L'Harmattan.

Charaudeau Patrick (1993), « Le contrat de communication dans la situation classe », J.-F. Halté, Inter-Actions, Université de Metz, 1993, consulté le 10 novembre 2015 sur le site de Patrick Charaudeau - Livres, articles, publications. www.patrick-charaudeau.com/Le-contrat-de-communication-dans.html.

Charaudeau Patrick (2014), «Étude de la politesse, entre communication et culture », A. M. Cozma, A. Bellachhab et M. Pescheux (dir.), Du sens à la signification: de la signification au sens. Mélanges offerts à Olga Galatanu, Berne, Peter Lang, 137-154.

Collette Karine (2007), "La concurrence discursive : analyse des logiques de discours dans la communication épistolaire adressée par lradministration publique aux usagers », Communication : information, médias, théories, pratiques, vol. 25, n², 107130.

Collette Karine (2013), « Distances et conciliations sur le statut du discours : autour d'une analyse émergente des discours du Printemps érable », Cahiers de recherche sociologique, n 54, 71-94.

Cozma Ana Maria (2009), Approche argumentative de la modalité aléthique dans la perspective de la Sémantique des Possibles Argumentatifs. Application au discours institutionnel de la bioéthique, Thèse de doctorat, Université de Nantes.

Culpeper Jonathan (2011), Impoliteness. Using language to cause offence, Cambridge, Cambridge University Press.

Damasio Antonio R. (1994), Descartes'Error. Emotion, Reason, and the Human Brain, New York, A. Grosset/ Putnam Books. 
Deshaies Denise et Diane Vincent (dir.) (2004), Discours et constructions identitaires, Québec, Presses de l'Université Laval.

Dorais Louis-Jacques (2004), «La construction de l'identité », D. Deshaies et D. Vincent (dir.), Discours et constructions identitaires, Québec, Presses de l'Université Laval, 111.

Dubar Claude ([1991] 1995), La Socialisation. Construction des identités sociales professionnelles, Paris, Armand Colin.

Dubar Claude (1996), «Usages sociaux et sociologiques de la notion d'identité », Formation et dynamiques identitaires. Education Permanente, $n^{\circ} 128,37-44$.

Ducrot Oswald (1984), Le Dire et le dit, Paris, Editions de Minuit.

Ducrot Oswald (1995), Topoï et formes topiques », J.-C. Anscombre (dir.), Théorie des topoï, Paris, Kimé, 85-100.

Galatanu Olga (1996), « Analyse du discours et approche des identités », Formation et dynamiques identitaires. Education Permanente, n’ 128, 45-62.

Galatanu Olga (2000), «Langue, discours et systèmes de valeurs », E. Suomela-Salmi (dir.), Curiosités linguistiques, Université de Turku, 80-102.

Galatanu Olga (2006), « Sémantique des possibles argumentatifs et dénomination », M. Riegel, C Schnedecker, P; Swiggers, I. Tamba (dir.), Au carrefour du sens. Hommages offerts à Georges Kleiber, Leuven, Peeters Publishers, 499-510.

Galatanu Olga (2009a), « Semantic and discursive construction of identites. "Europe of knowledge" in the academic discourse », E. Suomela-Salmi et Fred Dervin (dir.), Cross-linguistic and Cross-Cultural Perspectives on Academic Discourse, Amsterdam/Philadelphia, John Benjamins Publishing Company, 2009, 275-293.

Galatanu Olga (2009b), «L'Analyse du Discours dans la perspective de la Sémantique des Possibles Argumentatifs : les mécanismes sémantico-discursifs de construction du sens et de reconstruction de la signification lexicale », N. Garric, J Longhi (dir.), L'Analyse linguistique des corpus discursifs : des théories aux pratiques et des pratiques aux théories, Les Cahiers $L L L, n^{\circ} 3$, Clermont-Ferrand, Presses Universitaires Blaise Pascal, 49-69.

Galatanu Olga (2010a), "La construction discursive de la francophonie: sens, valeurs et images identitaires », L. Hébert, L. Guillemette (dir.), Performances et objets culturels, Québec, Presses de l'Université Laval, 207-223.

Galatanu Olga (2010b), «Pour une approche sémantico-discursive du concept d'identité : faute, crime et dynamique discursive », M. Palander-Collin, H. Lenk, M. Nevala, P. Sihvonen et M. Vesalainen (eds), Constructing Identity in interpersonal communication, Mémoires de la Société Néophilologique de Helsinki, LXXXI, 125-138.

Galatanu Olga (2013), «Introduction à l'étude du concept et de la signification lexicale de francophonie. Construction discursive d'un concept, activation d'un lien dénominatif, ou désignation d'un "objet social” ? », O. Galatanu, A. M. Cozma, V. Marie (dir.), Sens et signification dans les espaces francophones. La construction discursive du concept de francophonie, Berne, Peter Lang, 15-40. 
Galatanu Olga, Cozma Ana-Maria et Fravalo Loïc (2014), "Les valeurs sémantico-pragmatiques de "je t'explique", "je m'explique" et les représentations sémantiques des verbes "expliquer" et "a explica” ", A. Gautier, L. Pino-Serrano, C. Valcarcel Riveiro, D. Van Raemdonck (dir.), Complémentations, Berne, Peter Lang, 277-293.

Garric Nathalie (1999), "La médiation, entre connaissance de la discursivité et pratique discursive », N. Garric et J. Longhi (dir.), L'analyse linguistique des corpus discursifs : des théories aux pratiques et des pratiques aux théories, Les Cahiers LLL, n’3, Clermont-Ferrand, Presses Universitaires Blaise Pascal, 93-114.

Kleiber Georges (1984), « Dénominations et relations dénominatives », Langages, n 76, 7794.

Kleiber Georges (2001), «Remarques sur la dénomination », Cahiers de Praxématique, n³6, 2141.

Kleiber Georges (2003), «Sur la sémantique de la dénomination », Verbum, vol. XXV, n¹, 97-107.

Langacker Donald (2008), Cognitive Grammar. A Basic Introduction, Oxford, Oxford University Press.

Letourneau Jocelyn (dir.) (1994), La Question identitaire au Canada francophone. Récits, parcours, enjeux, hors-lieux, Sainte-Foy, PUL (coll. «Culture française d’Amérique »), VII-XVI.

Lutz Catherine (1982), «The domain of emotion words on Ifaluk », American Ethnologist, 9, 113-128.

Longhi Julien (2011), Visées discursives et dynamiques du sens commun, Paris, L’Harmattan.

Palander-Collin Minna, Hartmut Lenk, Nevala Minna, Païvi Sihvonene et Vesalainen Marjo (eds) (2010), Constructing Identity in Interpersonal Communication/Construction identitaire dans la communication/ Interpersonnelle/identitätskonstuktion in der interpersonalen Kommunikation, Mémoires de la Société Néophilologique de Helsinki, LXXXI.

Pascual Amparo (1997), « Le sujet comme processus inachevé », G. Bajoit et E.Belin (dir.), Contributions à une sociologie du sujet, Paris, L'Harmattan, 95-112.

Paveau Marie-Anne (2010), «Propositions critiques en philosophie du discours », Semen, n²9, 127-146.

Prieto Guzman Juan Pablo (2015), Analyse sémantique et discursive de représentations sur l'identité dans la réception du discours pédagogique. L'incidence interdiscursive, thèse de doctorat, Université de Nantes.

Ricour Paul (1990), Soi-même comme un autre, Paris, Seuil.

Riley Philip (2007), Language, Culture and Identity. An Ethnolinguistic Perspective, Londres, Continuum.

Sarfati Georges-Elia (2008), «Pragmatique, linguistique et normativité : remarques sur les modalités discursives du sens commun », Langages, $n^{\circ} 170,92-108$.

Siblot Paul (1997), « Nomination et production de sens : le praxème », Langages, n 127, 3855.

Siblot Paul (2001), «De la dénomination à la nomination. Les dynamiques de la signifiance nominale et le propre du nom », Cahiers de praxématique, n’36, 189-214.

Tracy Karen (2002), Everyday Talk. Building and Reflecting Identities, New York, Londres, The Guilford Press.

Veniard Marie (2013), La Nomination des événements dans la presse. Essai de sémantique discursive, Besançon, Presses Universitaires de Franche-Comté. 
Vincent Diane (2000), « L'argumentation et la construction de l'identité et de l'image des locuteurs. Une étude de cas: comment peut-on dire que l'on est heureux », G. Martel (dir.), Autour de l'argumentation: rationnaliser l'expérience quotidienne, Québec, Nota Bene, 127154.

\section{Corpus de textes étudiés}

Furio Jennifer (1998), The Serial Killers Letters. A Penetrating Look Inside the Minds of Murders, Philadelphia, The Charles Press, Publishers. 